\title{
Consumer behaviour and a healthy diet: a challenge or an opportunity?
}

\author{
Fereidoon Shahidi
}

Functional Foods and Nutraceuticals Laboratory, Department of Biochemistry, Memorial University of Newfoundland, St. John's, NL, Canada A1B 3X9, Canada. E-mail: fshahidi@mun.ca

DOI: $10.31665 /$ JFB.2021.15276

Received: September 28, 2021; Revised received \& accepted: September 28, 2021

Citation: Shahidi, F. (2021). Consumer behaviour and healthy diet: a challenge or an opportunity? J. Food Bioact. 15: 1-2.

\begin{abstract}
Healthy food choices by consumers are dictated by several factors but most would like healthy and affordable supply. Of course, traditional foods that are familiar to the consumers and have the appropriate sensory characteristics are most desirable to enhance immunity. Cultural background of the consumers often dictates their food habits and availability of local foods, driven by economic factors, are important considerations. Food safety, sustainability and traceability along with regulatory issues are to be considered in order to use the opportunities to address various challenges.
\end{abstract}

Keywords: Consumer behaviour; Healthy food; Sustainability; Traceability; Functional foods; Food safety; toxins; Health claims; Bioactives.

The International Academy of Food Science and Technology (IAFoST) and International Union of Food Science and Technology (IUFoST) hosted a round-table to discuss the consumer perception, behaviour, food habits, cultural background, gender, socioeconomic, food choices and dietary patterns and their influence on a healthy diet. The round Table was co-chaired by Dr. Aman Wirakartakusumah and Dr. Ugugua Charles Aworh. The rapporteurs were Dr. Chin-Kun Wang and Dr. Jairo Romero.

There were 5 panel members who spoke on different aspects of the importance of consumer behaviour on developments related to a healthy diet. Lifestyle and balanced diet, sustainability, nutrition, and functional foods were considered as important aspects. In addition, safety and consumer demands were considered as being components that should help in providing evidence-based and transparent information. The importance of education, cultural behaviour and food literacy in healthy food choices should also be considered. Attention to food loss and waste, local access and availability, role of food science and technology, incentives on healthy diet, and finally value addition and circular economy in food industry were considered important.

The first panel member was Professor Sir Charles Godfray of Oxford University. He discussed the environmental and health issues related to livestock and the fact that the largest green house emission, i.e., methane, is due to ruminants and their feed. He noted that for environmental issues, animal welfare, health, and consumers are factors needed for consideration as well as production cost and legal aspects.

The second panel member was Dr. Pavinee Chinachoti, chair of Food Innovation and Regulation Network from Thailand. She highlighted gaps in understanding safety and health claim regulations and that the food reaching the consumer must be safe and healthy. Product innovators must pay attention to the value chain. In this regard, to innovate, regulation should be recognized along with scientific developments, whilst health claims and functional food innovation roadmap must consider the fact that consumers are interested in transparency, traceability and more advanced food safety considerations that require appropriate technology and regulation in the supply chain.

The third speaker was Professor Sebastiano Porretta of the Ministry of Economic Development from Italy. He discussed consumer based new healthy food development. In this, he emphasized that consumers are demanding clean label products with very few lists of ingredients and no use of unfamiliar chemical names, especially of synthetic compounds (added by the author). Thus, attention needs to be paid to the paradigm shift. Consumers are interested in reasons for performance of products. In this regard, functional foods and ingredients from industrial wastes and process stream might lead to consumer preference because these 
co-products often have much higher concentration of bioactive compounds (added by the author). He noted that conjoint analysis provides a best approach. He also discussed segmentation and use of orthogonal functional design. In this regard, he noted the importance of natural, generated or induced compounds, price, sustainability, consumer preference, claims and label issues. Although it might be said that the consumer is ready to pay extra for healthy functional foods, in practice they often look for cheap and healthy food options.

The next speaker was Dr. Caroline Smith DeWaal, Global Alliance for Improved Nutrition. She talked about food safety and consumer demand and new tools for achieving development. She noted that 600 million food borne illnesses occur each year, thus ensuring access to safe and nutritious food is important. These result in 420,000 deaths each year and 125,000 of them are children of less than 5 years. These are due to microbial, helminths, aflatoxin, and other toxins. While $41 \%$ of world population is in Asia and Africa, $75 \%$ of such deaths is there. In addition, while $9 \%$ the population there is from children under $5,30 \%$ of deaths is in this age group. While access to safe and nutritious food is necessary, consumers are a major driving force. Establishing a functional knowledge platform to share information with all is essential so that progress could be made without duplication.

The last speaker was Dr. Petra Klassen Wigger, Global R \& D Scientific Advisor for Nutrition and Health of Nestle. She noted that leveraging science and technology to address challenges of sustainable healthy diet was desired as consumers demand food and beverages that are safe and healthful from a nutritional and ingredient standpoint and are respectful of the environment. They also want food and nutrition security for the present and for future generations. In this regard, climate change is putting pressure on food systems and the need for affordable food with consideration of sustainability warrants timely attention. Consumers are also able to recognize healthy foods and distinguish them from less desirable choices. However, some problems persist. These are related to the lack of understanding of how to achieve a balanced diet and nutritional risk when following a special diet, such as vegan food. Lack of knowledge to manage portion and frequency of consumption as well as awareness of micronutrients and benefits of fortification remain to be a challenge for most. Finally, misinterpretation of processing and its effects on food wholesomeness is an issue, especially for the ready-to-eat foods. In addition, understanding the local needs and attention to carbon footprint might be a consideration (from the author)

With regard to formulation and re-formulation, positive aspects related to micronutrients, protein, fiber, whole grain, nuts, fruits, vegetables and healthy oils as well as negative features related to sugar, sodium and salt content as well as trans fats (from the author) and energy content must all be considered when addressing dietary needs. To achieve these goals, a multi-disciplinary approach is needed to ensure science-based innovations. It is also essential to pay attention to the risk of exposure to plant toxins, mycotoxins, co-harvest issues, climate change, risk of adulteration, allergens and cross reactivity and cross contamination.

Overall, one might conclude that affordable nutrition, attention to raw material and loss upon harvest, storage and transportation or processing waste, use of innovative technologies, including fermentation, as well and use of adequately processed food are essential in addressing consumer's needs. To achieve desirable sensory character fermentation and generation of a sour taste, for example, may help in better appreciation of formulations that might otherwise be unfamiliar to the consumers. While there are many challenges, there are also opportunities that we must take advantage of. In this regard, climate change and growing world population require urgent actions to ensure sustainability, healthfulness, and affordable diet. Science based innovation is a key driver and may constitute a shift to a plantbased diet, avoiding food loss and valorize side streams. Therefore, a holistic approach to ensure various aspects of consumer's diet for better health and wellbeing of ecosystem that supports healthier society, and the planet, must be considered. 